\title{
Arte e tabù: il disgusto e i limiti della rappresentazione
}

\author{
di Serena Feloj \\ serena.feloj@unipv.it
}

\begin{abstract}
Referring to the notion of representation, I will argue the idea that disgust continues to constitute a limitation for art and that the presence of the disgusting can be read through the Freudian notion of taboo. The indulgence of artistic creation in disgust, in fact, would confirm the fact that only what is domesticated, thus what does not arouse authentic disgust, can be assimilated by art. On the contrary, authentic disgust remains taboo insofar as it indicates the absolutely other, the unassimilable, a non-domesticated animality extraneous to the process of civilization. It is, after all, what classical aesthetics had already shown and that today can be reread in Freudian terms.
\end{abstract}

Keywords: Disgust, Taboo, Art, Representation, Limits

In un recente saggio, breve ma di tutt'altro che agile lettura, il critico d'arte Jean Clair si interroga sulla presenza del disgustoso nell'arte contemporanea e registra che «mai l'opera d'arte è stata così cinica e ha così amato sfiorare la scatologia, la lordura e l'oscenità. E mai - fatto ancor più sconcertante quest'opera è stata così prediletta dalle istituzioni»' ${ }^{1}$. Non è difficile, infatti, trovare esempi di opere d'arte, siano esse visive o letterarie, che costringono il fruitore a fare i conti con materiali disgustosi e immagini ributtanti. Da Diane Arbus a Hermann Nitsch, da Cindy Sherman sino a Damien Hirst, sono numerosi gli esempi di opere che, in maniera differente, possono essere e sono state ricondotte al disgusto. Questa massiccia presenza di elementi disgustosi nelle opere d'arte, quali cadaveri, materie organiche di scarto e secrezioni, chiama l'estetica e la filosofia dell'arte a rendere conto di un movimento che

1 J. Clair, De Immundo (2004), tr. it. di P. Pagliano, Abscondita, Milano 2016, p. 23.

Materiali di Estetica - N. 8.2: 2021, Pagina 326 
investe la società stessa. Se, infatti, con Freud ${ }^{2}$, si vuole intendere l'arte come la rappresentazione più elevata di quel processo di addomesticamento delle pulsioni e degli istinti, in sintesi del processo di civilizzazione, viene da chiedersi come leggere questa paradossale presenza di ciò che ha carattere corporeo, istintuale e pulsionale nel prodotto della più alta forma di civilizzazione, di educazione e di cultura, ossia nell'opera d'arte.

Ciò che proverò a sostenere è l'idea che il disgusto continui a costituire un limite per la rappresentazione artistica e che la presenza del disgustoso in arte può essere letta attraverso la nozione freudiana di tabù. L’indulgere della creazione artistica nel disgustoso, infatti, non farebbe altro che confermare il fatto che soltanto ciò che viene addomesticato, dunque ciò che non suscita un autentico disgusto, può essere assimilato dall'arte. Il disgusto autentico rimane invece tabù in quanto indica l'assolutamente altro, l'inassimilabile, un'animalità non addomesticabile estranea al processo di civilizzazione. È del resto quanto già l'estetica classica aveva mostrato e che oggi può essere riletto in termini freudiani.

\section{Gusto e disgusto}

Sono infatti convinta che le categorie dell'estetica permettono di elaborare una risposta filosofica capace di leggere la presenza del disgustoso nell'arte e di restituire questo fenomeno in tutta la sua complessità. $\mathrm{Ci}$ si potrebbe limitare a leggere nei fenomeni artistici disgustosi la ricerca da parte dell'arte di stimoli sempre nuovi e sempre più violenti. Ma ritengo che questa sia soltanto una parte della risposta che la filosofia può dare oggi. Nella storia del pensiero, e dell'estetica in particolare, la categoria del disgusto nasce con lo stesso sorgere della società del gusto, nel Secolo dei Lumi, ma non può essere definita in termini semplicemente oppositivi. Gusto e disgusto sono,

\footnotetext{
${ }^{2}$ Cfr. S. Freud, Sigmund, Contributi alla psicologia della vita amorosa, in Opere 1909-1912, tr. it. a cura di C. L. Musatti, Bollati Boringhieri, Torino 1989.
} 
infatti, profondamente connessi tra loro e la definizione dell'uno contiene la definizione dell'altro. Anche dal punto di vista teorico, a prescindere dal suo sviluppo storico, la nozione di disgusto chiama a restituire un quadro complesso: il disgusto è infatti una reazione anzitutto fisiologica, in grado però di avere anche una declinazione morale. Si tratta di una risposta emotiva, solo apparentemente riferibile a dinamiche naturali, dotata di un carattere profondamente culturale.

L'ambiguità entro cui si muove il disgusto, tra bisogno e sazietà, rivela il proprio carattere problematico se si pensa al termine tedesco impiegato fin dal Settecento per indicare la categoria del disgusto: Ekel, che indica sia un rifiuto assoluto e irredimibile, sia la nausea per eccessiva sazietà ${ }^{3}$. Non è secondaria questa ambiguità lessicale per la costituzione di un ben delimitato, e decisivo, dibattito sul disgusto che è da collocarsi in Germania a partire dagli anni Cinquanta del Settecento. La questione che muove questo dibattito è se l'arte sia legittimata a rappresentare il disgustoso. La discussione di questo problema porterà una definizione del bello, dell'opera d'arte e, infine, dell'estetica e dei suoi limiti.

In un contesto di definizione dell'estetica come nuova disciplina a seguito del testo di Baumgarten del 17504, secondo Moses Mendelssohn il disgusto è anzitutto dovuto a un'eccessiva sazietà, al troppo dolce e abbondante; sarà questa riflessione che darà avvio all'elaborazione, da parte di Mendelssohn, della teoria dei sentimenti misti. Con ci Mendelssohn sostiene la necessità di definire sensazioni che accostino al piacevole il suo opposto, il dispiacere, al fine di ottenere maggiori durata e incisività.

L'ottantaduesima Lettera sulla letteratura di Mendelssohn afferma che il disgusto sorge anzitutto dalla dolcezza eccessiva: il gusto del dolce viene

\footnotetext{
${ }^{3} \mathrm{Nel} \mathrm{Grimm} \mathrm{Wörterbuch,} \mathrm{compare} \mathrm{immediatamente} \mathrm{l'associazione} \mathrm{del} \mathrm{termine} \mathrm{Ekel} \mathrm{con}$ "fastidium, taedium, nausea», che indica sia la comunanza tra disgusto e sazietà, sia quella tra disgusto e noia.

${ }^{4}$ Cfr. A.G. Baumgarten, L'Estetica (1750), tr. it. a cura di S. Tedesco, Aesthetica, Palermo 2000 .
} 
paragonato alla bellezza e il disgusto insorge proprio quando il dolce o il bello diventano eccessivi e sono, afferma Mendelssohn, «troppo puri». Il disgusto dunque sopraggiunge qualora il bello o il dolce risultino stucchevoli. Su questa idea viene fondata la teoria del sentimento misto: affinché divenga una reale fonte di piacere e per non suscitare disgusto, il bello deve sempre trovarsi mescolato a un elemento orrido, sublime o terrificante. Secondo Mendelssohn il disgusto è, quindi, parte integrante della stessa categoria della bellezza, che può sempre rovesciarsi nel suo opposto proprio a causa della sua purezza.

In linea con Mendelssohn, Lessing colloca nel disgusto il limite dell'arte: il disgusto è il pericolo maggiore per l'esibizione artistica non semplicemente perché suscita un sentimento sgradevole nello spettatore, ma specialmente perché l'arte cessa di essere tale qualora susciti disgusto. Ciò non significa che non possa rappresentare il disgustoso; il suo limite è, piuttosto, indicato nella reazione del fruitore, che può certo provare sensazioni spiacevoli di fronte all'opera d'arte, ma la sua risposta emotiva non può essere di totale rifiuto.

La riflessione di Lessing sulla relazione tra arte e disgusto si colloca nel cuore dell'estetica classica e restituisce una dinamica che può essere impiegata anche nel dibattito contemporaneo, come propone di fare Jean Clair. Il dibattito sul disgusto che si sviluppa in Germania a metà del Settecento, qualora assunto nella sua complessità teorica, dimostra, infatti, l'impossibilità da parte dell'arte di suscitare un'autentica reazione di disgusto. In tal senso l'estetica classica tedesca fornisce ancora oggi argomentazioni valide per sostenere la tesi freudiana secondo la quale il disgusto costituisce una forma di tabù: nemmeno l'arte, che si è detto essere l'espressione più elevata del processo di civilizzazione e forma di legittimazione delle trasgressioni di questo processo, può realizzare un superamento del disgusto. 
È nel dibattito tedesco sul disgusto che si attesta quindi l'idea, espressa chiaramente da Kant a fine secolo, per cui: «solo una specie di bruttezza non può venire rappresentata in modo conforme alla natura senza demolire ogni compiacimento estetico e dunque la bellezza artistica: quella, cioè, che suscita disgusto» ${ }^{5}$. Ponendo le basi per quanto accadrà nei secoli successivi, già nel XVIII secolo all'arte viene attribuita una capacità di «addomesticamento», in grado di restituire in maniera assimilabile anche ciò che nella realtà risulta insopportabile. Il disgusto rimane così a segnare il confine con ciò che è assolutamente inassimilabile.

Secondo Herder, la "natura stessa» non ha "dato a nessuna sensazione spiacevole una sfera così ristretta come al [...] disgusto»; ma di gran lunga ancora più ristretta è questa sfera del disgusto nell'arte. Herder sottolinea la rarità, addirittura la non esistenza del disgusto, che è escluso dall'estetica, al punto da formulare persino l'idea di un fantasma del «disgusto reale» ${ }^{6}$. Il disgusto quindi può essere suscitato tramite l'opera d'arte soltanto come derivato, come «associazione» non autentica, come trascrizione metaforica: in definitiva il disgusto non può entrare a far parte del campo dell'arte.

Ciò che rende il disgusto il tabù per eccellenza nella rappresentazione artistica è da ricondurre a due caratteristiche. Il disgusto è, già per Mendelssohn, essenzialmente legato ai «più oscuri di tutti sensi», all'olfatto, al tatto e al gusto. La sensazione di disgusto non può quindi abitare, se non per associazione, in un'esperienza, quale è quella artistica, che è anzitutto visiva e uditiva; o, altrimenti detto, che è il prodotto più elevato del processo di civilizzazione e, soltanto mediatamente, cioè attraverso un godimento anzitutto intellettuale, può richiamare lo stato di animalità al di sopra del quale ci ha elevati.

\footnotetext{
${ }^{5}$ I. Kant, Critica della capacità di giudizio (1790), tr. it. a cura di L. Amoroso, Rizzoli, Milano 1995 , p. 439.

${ }^{6}$ J.G. Herder, Erstes Kritisches Wäldchen, in Schriften zur Ästhetik und Literatur 1767-1781, hrsg. von G.E. Grimm, Deutscher Klassiker Verlag, Frankfurt am Main 1993, p. 242.
} 
La seconda caratteristica essenziale del disgusto, sempre a partire dal dibattito settecentesco, ha a che fare con la distinzione tra natura e arte. Per gli autori dell'estetica classica tedesca, l'opera d'arte è anzitutto una forma di illusione: per Lessing, il poeta deve farci «credere» di «vedere» immediatamente Elena o lo scudo di Achille7, la rappresentazione artistica deve realizzare un complesso equilibrio tra naturalezza apparente e costruzione illusoria ${ }^{8}$. L'illusione estetica è possibile poiché l'arte si pone come medium che permette uno sguardo privilegiato e inquadrato entro una cornice sulla realtà. Il disgusto è invece una reazione senza mediazioni: è un rifiuto immediato, che non ammette redenzioni, che si attiva di fronte a un oggetto reale e realmente ripugnante. Se quindi l'arte confonde i piani della realtà e dell'illusione, il disgusto è una reazione violenta che fa cadere questo equilibrio. Così se l'arte generasse una reazione di autentico disgusto, cesserebbe di essere illusione per divenire realtà; e con ciò non sarebbe più arte. È questo un altro tratto di ambiguità del disgusto che permette di definire questa categoria come il tabù dell'arte.

\section{L'abietto}

In tal senso ritengo si possa sostenere ancor oggi che la sensazione di disgusto continua a indicare, al di là della legittimazione del disgustoso in arte, qualcosa di inassimilabile, qualcosa di assolutamente altro che rimane inaccessibile all'arte. Il disgusto, come reazione totalmente negativa, assume quindi una funzione discriminatoria e di barriera difensiva innalzata dal processo di civilizzazione. L'idea del disgusto come tabù dell'arte ha poi trovato un'interessante eco nella contemporaneità tramite la nozione di

\footnotetext{
${ }^{7}$ Cfr. G.E. Lessing, Laocoonte (1766), tr. it. a cura di M. Cometa, Aesthetica, Palermo, 2000, pp. 63-89; I. Mülder-Bach, Im Zeichen Pygmalions. Das Modell der Statue und die Entdeckung der "Darstellung" im 18. Jahrhundert, Fink, München, 1998, pp. 103-148.

8 Cfr. S. Feloj, Estetica del disgusto. Mendelssohn, Kant e i limiti della rappresentazione, Carocci, Roma 2017, pp. 85-90.
} 
«abietto» che, soprattutto a partire dagli anni Ottanta del Novecento, ha spinto l'arte a confrontarsi nuovamente con i propri limiti. Il saggio di Julia Kristeva sull'abiezione ${ }^{9}$ richiama l'attenzione sulla relazione tra oggetto e soggetto che caratterizza essenzialmente il disgusto.

La reazione di disgusto, diversamente da altre emozioni negative come ad esempio la vergogna, è per lo più rivolta verso l'esterno e sta a indicare l'impossibilità di assimilare, di fare propria, un'alterità assoluta. L'abietto è quindi ciò che, colpito da disgusto, è da rigettare, al punto che non può essere assimilato, nemmeno tramite il linguaggio o l'immaginazione: è l'innominabile e l'irrappresentabile. L'abietto non è tuttavia identico al disgusto. In entrambi i casi si tratta di qualcosa che il soggetto nauseato rigetta, percependo la presenza dell'oggetto come una minaccia. In entrambi i casi si ha a che fare con un rimosso, con la dimensione più animale della nostra esistenza che è stata resa tabù. E in entrambi i casi, come ha mostrato Freud $^{10}$, il rifiuto che viene espresso è percorso da una certa forma di ambiguità, laddove la ricerca di ciò che genera avversione diviene manifestazione della perversione. Abiezione e disgusto sono, tuttavia, essenzialmente differenti dal momento che l'abietto può trovare una redenzione, che invece è negata al disgusto. Questa differenza è ancora da ricondursi anzitutto al fatto che l'abietto designa un oggetto, mentre il disgusto indica sempre lo stato d'animo di un soggetto.

L'abiezione elabora "un trivio di fobia, di ossessione e di perversione» ${ }^{11}$ che dà luogo a una «jouissance perverse» ${ }^{12}$ : è una sublimazione dell'abiezione sotto forma di riconciliazione con tutto ciò che è stato rifiutato. E questa riconciliazione è resa possibile, secondo Kristeva, proprio tramite l'arte.

\footnotetext{
9 J. Kristeva, Poteri dell'orrore. Saggio sull'abiezione (1980), tr. it. a cura di A. Scalco, Spirali, Milano 1981.

${ }^{10}$ Cfr. W. Menninghaus, Disgusto. Teoria e storia di una sensazione forte, a cura di S. Feloj, Mimesis, Milano 2016, pp. 255-306.

11 J. Kristeva, Poteri dell'orrore, cit., p. 51.

12 Ivi, p. 243.
} 
La capacità simbolica dell'arte è infatti in grado di restituire l'abietto che è stato rifiutato. Kristeva attribuisce senz'altro un ruolo decisivo alle strutture simboliche dell'arte, che restituiscono l'essere reale e la verità. Questa concezione dell'arte, senz'altro non unica nel suo genere, viene recepita dal mondo artistico, che sempre più si confronta con oggetti di scarto, di rifiuto, vale a dire con i fenomeni dell'abiezione. Questo confronto non è, tuttavia, una riabilitazione del disgusto, né supera i confini dell'estetica tracciati al momento della sua nascita nel XVIII secolo. L'assimilazione dell'abietto tramite l'arte costituisce, infatti, un passo ulteriore sullo stesso percorso, inaugurato da Mendelssohn e da Lessing, di legittimazione del brutto e poi del disgustoso. Si tratta cioè pur sempre di un «addomesticamento».

L'assimilazione dell'abietto in arte e in letteratura diviene un'appropriazione simbolica della realtà che è oggi definita come Abject Art, Post-human Art o arte post-organica: il corpo, in particolare quello dell'artista stesso, subisce delle trasformazioni, divenendo esso stesso l'oggetto dell'opera d'arte. Lea Vergine, nel suo libro Body Art e storie simili, afferma che «l'artista diventa il suo oggetto. Meglio, l'artista è tetico di sé ed è tetico dell'oggetto, pone cioè se stesso come oggetto, essendo cosciente di tale processo» ${ }^{13}$. Alla base di questi fenomeni artistici è possibile intravedere un ritorno al reale, senza però offrire ai sensi di chi osserva alcun filtro, dando in questo modo una dimostrazione della realtà stessa. Ma la concessione di questa verità è data dall'arte abietta in modo prepotente, con una violenza traumatica sia per lo spettatore sia per l'artista stesso. Si tratta di un «realismo traumatico» ${ }^{14}$ che caratterizza l'arte della stagione abietta:

\footnotetext{
${ }^{13}$ L. Vergine, Body Art e storie simili. Il corpo come il linguaggio, Skira editore, Milano 2000, p. 15.

${ }_{14}$ G. Patella, "Tra Abiezione e Disgusto. Il corpo ferito dell'arte contemporanea", Horti Esperidum, I, 2016, pp. 281-322.
} 
degli anni Novanta dei vari Paul McCarthy, Kiki Smith, Carolee Schneemann, Cindy Sherman, Damien Hirts ecc., in cui viene in primo piano l'inscrizione delle forme dell'arte nelle trame conturbanti di corpi feriti, spezzati, degradati e disfatti, spinti nei territori dell'abiezione, dell'informe e del disgusto, ridotti semplicemente a oggetti di scarto, residui o escrementi ${ }^{15}$.

Con l'arte abietta si riapre così la stessa questione che aveva animato il dibattito di metà Settecento intorno alla tragedia: che cosa attira gli spettatori alla visita delle numerose esibizioni di arte abietta? Perché il pubblico ne è istintivamente inorridito e allo stesso momento affascinato? Non è semplicemente, o soltanto, una ricerca di innovazione continua, di trasgressione e di shock sempre più forti, dal momento che questa sola intenzione spesso va incontro all'abitudine agli eccessi che sfocia nell'ironia e nell'indifferenza. Occorre piuttosto ricorrere alla natura simbolica dell'arte, al suo carattere illusorio di contro al reale per intendere queste forme d'arte come una restituzione addomesticata e sopportabile dell'abietto. Si tratta allora di una purificazione dell'impuro.

Per Kristeva, l'abiezione è sempre congiunta alle istituzioni religiose ed emerge in concomitanza con il loro crollo:

\footnotetext{
le diverse modalità di purificazione dell'abietto - le diverse catarsi - costituiscono la storia delle religioni e si compiono nella catarsi per eccellenza che è l'arte, al di qua e al di là della religione. Vista in quest'ottica l'esperienza artistica, la quale si radica nell'abietto e parlandone lo purifica, risulta una componente essenziale della religiosità. Forse per questo è destinata a sopravvivere al crollo delle forme storiche delle religioni ${ }^{16}$.
}

L'arte, come catarsi per eccellenza, realizza una mediazione che permette un'identificazione mimetica con ciò che provoca ribrezzo e fastidio e soltanto in questo modo è possibile regredire e incarnare l'oggetto, che così si eleva nuovamente. Gli eccessi di sostanze escrementizie o pezzi di organismi, anche umani, che dagli anni Ottanta sono presenti nei musei e nelle gallerie d'arte di tutto il mondo sono le testimonianze di questo processo di purificazione.

\footnotetext{
15 Ibidem.

16 J. Kristeva, Poteri dell'orrore, cit., p. 19.
} 


\section{L'immondo}

Esemplificativo dell'abiezione, intesa secondo la teoria di Kristeva, è il cadavere. Il corpo privo di vita, o persino in decomposizione, è espressione della duplicità umana e diviene simbolo della contaminazione fondamentale alla quale la vita, in quanto uomini, ci espone. Nella storia delle religioni, il cadavere è ciò che deve essere escluso da tutto quello che è vita e non deve avere la possibilità di contaminare la terra divina. Il corpo morto e in deperimento sta a indicare tutto ciò che non è spirituale, simbolico e legge divina. A questa dimensione dell'abiezione si possono ricondurre alcune opere di Damien Hirst ${ }^{17}$, che nel 1993 espone il suo squalo in formaldeide, fermando il momento in cui un corpo vivo, pieno di spirito, passa a essere un elemento abietto, uno scarto, un non-oggetto. E sempre ai meccanismi dell'abiezione è possibile ricondurre il successo che da anni continua a suscitare la mostra Body World, di Gunther von Hagens, con cadaveri umani in esposizione.

La teoria dell'abiezione permette di leggere questi fenomeni dell'arte contemporanea, che si confronta con il corrotto e con il disgustoso, che esibisce l'impuro in ogni sua forma, come manifestazioni della necessità di spiritualità e di purificazione. Tanto più un corpo è trafitto, distrutto, degradato e privo di vita, tanto più esso celebra lo spirito vivente ed eterno. Questa teoria è di fatto una conferma della forza del tabù che governa l'arte. Il mondo dell'arte contemporanea rischia, tuttavia, spesso di dimenticare la complessità che ancora comporta l'esclusione del disgusto dall'esperienza artistica e l'inclusione del disgustoso e dell'abietto nel prodotto dell'arte. Jean Clair ha recentemente richiamato l'attenzione su ciò che la relazione tra arte e disgusto può significare per la nostra civiltà:

Non potevo immaginare che i musei, invece di essere un rifugio al riparo dal mondo moderno, significassero per me trovarmi nel cuore di un laboratorio dove

17 Cfr. il recente studio: M. Buelli, Un'estetica del disgusto. Damien Hirst, Galassia Arte, Milano 2017. 
meglio si leggevano i segni annuncianti il crollo della nostra cultura. Nel silenzio dei quadri, lontano dai tumulti del secolo, l'osservatore delle opere di cui avevo la cura, mi informava nel modo migliore sul lento processo di decomposizione di cui il nostro mondo è diventato preda. La storia dell'arte detta moderna era la storia della nostra propria fine. [...] Sintomi più evidenti di una adorazione dell'uomo da parte dell'uomo, che si conclude nella spazzatura e nell'imbecillità ${ }^{18}$.

Coniugando le categorie dell'estetica classica con la teoria freudiana che vede nel disgusto la manifestazione più alta del nostro processo di civilizzazione, Clair denuncia la situazione in cui versa il mondo attuale dell'arte in cui i musei non sono più strumenti della cultura, ma sono sottoposti principalmente al mercato e alla speculazione.

\begin{abstract}
Esponiamo quindi il vitello di Damien Hirst accanto a un'opera di Joseph Beuys, o meglio di Robert Morris, opere già accreditate, dotate di rating finanziario tripla o doppia nel mercato dei valori. Facciamolo poi entrare in un circuito di gallerie private, [...] con una bella sede e visibilità internazionale. [...] Gli azionisti che finanziano il progetto, portano i capitali e assumono i rischi, promettendo capitali. Infine [...] si otterrà da un'istituzione pubblica, un museo per esempio, l'organizzazione di una mostra dell'artista, di cui le spese tuttavia saranno coperte dalla galleria che lo promuove ${ }^{19}$.
\end{abstract}

Le riflessioni di Clair assumono una prospettiva del tutto originale qualora prendono in considerazione la relazione tra l'arte contemporanea e il disgusto. Clair richiama l'elemento di ambiguità che già per Freud caratterizza il disgusto a partire dal Parmenide di Platone. Nel testo platonico, «capelli, fango, sporcizia o altro privo di importanza e valore» ${ }^{20}$ sono definiti come sostanze che esistono solo nell'apparenza, che non partecipano di nessuna idea. Nella Repubblica, queste stesse sostanze che suscitano orrore muovono però anche il desiderio. Si tratta dell'ambiguità tra orrore e desiderio che spinge un uomo a guardare con ossessione una scena raccapricciante, pur sapendola tale. È l'ambiguità di sentimenti che coglie Leonzio di fronte all'abiezione del cadavere: non riusciva a distogliere lo sguardo da un mucchio

18 J. Clair, La part de l'ange. Journal 2012 - 2015, Gallimard, Paris, 2016.

19 A. Zaccuri, "La premiazione. Jean Clair, i titoli tossici dell'arte", Avvenire, 10 Maggio 2014.

20 Platone, Parmenide, 130 C, tr. it. di M. Migliori, in Platone. Tutti gli scritti, a cura di G. Reale, Bompiani, Milano, 2000. 
di cadaveri al di fuori dalle mura della città, seppur indignato dal suo stesso comportamento.

Consapevole di questa duplicità del sentimento, Clair rifiuta l'idea che un artista possa comporre arte con materiali abietti. Negli anni Novanta del secolo scorso, l'abject-art sembra però essere al culmine del successo: il 2 Ottobre 1992 al Castello di Rivoli viene inaugurata la mostra Post Human; nel 1993 al Whitney Museum of American Art di New York viene allestita una mostra dal titolo Abject Art. Repulsion and Desire; nel 1997 fece scalpore la mostra Sensation, alla Royal Academy di Londra, trasferita poi al Brookling Museum di New York nel 1999; nel 2000 l'opera di Tracey Emin My Bed viene insignita del Turner Prize, Grand Prix des Arts in Gran Bretagna.

Secondo Clair, l'arte dell'abiezione «sarebbe lo stato di un'arte inferiore, o anche di un'arte dei rifiuti, un'arte di ciò che resta dopo che tutto è stato rigettato» ${ }^{21}$, è la «morte clinica» ${ }^{22}$ dell'arte. Clair rinnova la questione che chiede cosa qualifichi la categoria del disgusto come tanto interessante per l'estetica; e si domanda che cosa renda possibile questo tipo di arte. Come è possibile che il pubblico voglia ammirare il letto sfatto e sporco di Tracey Emin, esposto in una delle più importanti istituzioni museali del mondo, oppure come possa sopportare le fotografie scattate furtivamente dentro gli obitori da parte di Andres Serrano o da Jeffrey Silverthorne?

Più inquietante della loro realizzazione, è l'accoglienza riservata a tali opere. [...] Tutto l'establishment del gusto pare applaudire quest'arte dell'abiezione. [...] Tutto avviene come se l'unità del socius, un tempo assunta dalla religione e dalla politica, ormai impossibilitate a mantenerla, avesse trovato la sua base nella manifestazione pubblica di una scatologia accettata e celebrata ${ }^{23}$.

Anche per Clair, come per Kristeva, l'arte si sostituisce alla religione e, con ciò, si misura con l'immondo, ossia con ciò che nega il mundus della legge

\footnotetext{
21 J. Clair, De Immundo, cit., p. 20.

22 Ibidem.

${ }^{23}$ Ivi, p. 23.
} 
religiosa. E l'artista diventa un sacerdos al servizio della nuova sacralità. La rinuncia alla religiosità tradizionale si configura come una trasgressione che rimane comunque entro i limiti del sacro, laddove il rifiuto di qualcosa è al tempo stesso l'affermazione della sua stessa esistenza. Clair propone come esempio quella che è forse la manifestazione più dissacrante dell'arte del Novecento: l'azionismo viennese. Le azioni di Hermann Nitsch o Otto Mühl, oltre a essere estremamente cruente, quasi animalesche, profanano i simboli religiosi, le funzioni corporali e le pratiche sessuali. Si distaccano dalla tradizione artistica dell'uso della tela o della scultura, le tempere vengono sostituite dal sangue che sgorga dalle ferite auto-inflitte, l'espressione avviene liberamente, attraverso il proprio corpo. I luoghi e i tempi delle opere, pur essendo del tutto sconvolti, tentano però di ricreare momenti rituali a scopo terapeutico e catartico.

In questa tipologia di creatività artistica, che si confronta direttamente con l'abietto, l'uomo diventa il soggetto stesso del sacrificio, ma questo individuo non è più un «portatore di cultura, bensì l'uomo animalizzato, la specie zoologica "uomo" ${ }^{24}$. Nella prospettiva di Clair, l'arte abietta mira a concretizzare la natura effimera della vita, a delimitare il tempo nella finitezza del qui e ora. Al tempo stesso, questo tipo di arte si appella a un indefinito e mitologico passato che diviene reliquia. Nella relazione tra mondo e immondo, tra arte e religione, tra dissacrazione e sacralità, per Clair la nozione di reliquia è centrale. Nella tradizione cristiana, le reliquie sono resti corporali, oggetti appartenuti a santi e beati, onorati in luoghi sacri attraverso un culto reliquiario che venera e rende partecipi di una «teologia dell'incarnazione e della resurrezione» ${ }^{25}$.

Un frammento sacro, però, ha dei tratti distintivi: anzitutto è tale ciò che del corpo del santo resta, in sostanza tutto quel che dopo la morte si fa residuo

\footnotetext{
24 Ivi, p. 59.

25 Ivi, p. 62.
} 
e che restituisce la sua vera identità di santo. Il resto degli umori che si possono identificare come lasciti di un corpo, la saliva, la bile o altre escrezioni, non sono invece reliquie. Non possiedono nulla di santo e non racchiudono nessuna concezione di sacrificio né, quindi, di santità. Nelle opere d'arte contemporanee, come nota criticamente Clair, la materialità con cui si vuole sostituire il culto religioso ha apparentemente a che fare più con scarti privi di significato che con la reliquia: sono solo scarti e umori che disorientano la natura stessa dell'opera, senza comprendere la sua stessa realtà. D'altra parte, scrive Clair, «i rituali degli azionisti, le manipolazioni della body art, [...] stanno in rapporto all'arte tradizionale - i quadri, le sculture - come la reliquia sta in rapporto con le immagini devote»26. Si è attuato un rovesciamento delle pratiche artistiche che ha accompagnato la fine dell'utilizzo delle arti figurative classiche, rinunciando al potere illusorio dell'arte, in favore di un uso del corpo nella sua concretezza nuda e cruda.

L'arte oggi ha rinunciato al desiderio di rappresentare la realtà del disegno e della pittura, ma, secondo Clair, accoglie ciò che tradizionalmente apparteneva alla pratica religiosa, ossia il culto delle reliquie: «nell'arte c'è un ritorno alla reliquia e alla presenza oggettiva del corpo e dei suoi umori, mentre allo stesso tempo la religione si è disincarnata ${ }^{27}$. L'ambivalenza tra sacro e dissacrazione che caratterizza l'arte abietta non soltanto fissa un'ulteriore caratteristica del disgusto come organo di distinzione tra puro e impuro. Le opere d'arte che indulgono su materiali abietti e disgustosi assumono significato unicamente perché si tratta di oggetti colpiti da disgusto e in questa forma di apparente violazione delle barriere che costituiscono la cultura e la civiltà occidentali, se ne conferma al tempo stesso l'esistenza. Ecco che il disgusto si conferma come tabù dell'arte. Nella dissacrazione artistica,

\footnotetext{
26 Ivi, p. 65.

${ }^{27}$ Ivi, p. 69.
} 
come forma di frantumazione del sacro, l'arte esprime il bisogno di superare il tabù del disgusto, ma al tempo stesso ne dimostra la forza.

Clair riprende la definizione dell'estetica classica per cui l'arte è sempre un'azione del «comporre»28; l'arte è una messa in forma. Secondo Clair, l'essere umano non è in grado di sopportare una decomposizione della forma, non è in grado di rappresentarsi una radicale assenza di forma. E nemmeno l'arte abietta costituisce un unicum; essa rappresenta invece lo sforzo, comune a ogni espressione artistica, di dare forma allinforme. La dissacrazione messa in atto dall'arte abietta è quindi soltanto un inganno: non vi è alcun superamento della distinzione tra puro e impuro, tra sacro e corrotto. Il disgusto continua a colpire ciò che non può essere assimilato ed è un tabù che non può essere superato. In questo senso l'opera d'arte rimane, come l'aveva definita Freud, una forma leggera di ipnosi che ci permette di riconciliarci con il mondo esterno.

\section{Nota bibliografica}

BAUMGARTEN, Alexander Gottlieb, Assthetica (1750), Olms, Hildesheim 1986; L'Estetica, tr. it. a cura di S. Tedesco, Aesthetica, Palermo 2000.

BUELLI, Manuela, Un'estetica del disgusto. Damien Hirst, Galassia Arte, Milano, 2017.

CLAIR, Jean, De Immundo, Édition Galilée, Paris 2004; De Immundo, tr. it. di P. Pagliano, Abscondita, Milano 2016.

—, La part de l'ange. Journal 2012 - 2015, Gallimard, Paris, 2016.

FELOJ, Serena, Estetica del disgusto. Mendelssohn, Kant e i limiti della rappresentazione, Carocci, Roma 2017.

${ }^{28}$ Ivi, p. 16. 
FREUD, Sigmund, "Über die allgemeinste Erniedrigung des Liebeslebens", in Gesammelte Werke, Fischer, Frankfurt am Main 1966-1969, Bd. 8; Contributi alla psicologia della vita amorosa, in Opere 1909-1912, tr. it. a cura di C.L. Musatti, Bollati Boringhieri, Torino 1989.

HERDER, Johan Gottfried, Erstes Kritisches Wäldchen, in Schriften zur Ästhetik und Literatur 1767-1781, hrsg. von G. E. Grimm, Deutscher Klassiker Verlag, Frankfurt am Main 1993.

KANT, Immanuel, Kritik der Urtheilskraft (1790), in Kant's gesammelte Schriften, hrsg. von der Königlich Preußischen Akademie der Wissenschaften, Georg Reimer, Berlin 1907 ss., Bd. 5; Critica della capacità di giudizio, tr. it. a cura di L. Amoroso, Rizzoli, Milano 1995.

KRISTEVA, Julia, Pouvoirs de l'Horreur. Essai sur l'Abjection, Seuil, Paris 1980; Poteri dell'orrore. Saggio sull'abiezione, tr. it. a cura di A. Scalco, Spirali, Milano 1981.

LESSING, Gotthold Ephraim, Laokoon: Oder über die Grenzen der Malerei und Poesie (1766), in Werke 1766-1769, hrsg. von W. Barner, Bd. 5/2, Deutscher Klassiker Verlag, Frankfurt am Main 1990; Laocoonte, tr. it. a cura di M. Cometa, Aesthetica, Palermo, 2000.

MENNINGHAUS, Winfried, Disgusto. Teoria e storia di una sensazione forte, a cura di S. Feloj, Mimesis, Milano 2016.

MÜLDER-BACH, Inka, Im Zeichen Pygmalions. Das Modell der Statue und die Entdeckung der "Darstellung” im 18. Jahrhundert, Fink, München, 1998.

PATELLA, Giuseppe, "Tra Abiezione e Disgusto. Il corpo ferito dell'arte contemporanea", Horti Esperidum, I, 2016, pp. 281-322. 
PLATONE, Parmenide, tr. it. di M. Migliori, in Platone. Tutti gli scritti, a cura di G. Reale, Bompiani, Milano, 2000.

VERGINE, Lea, Body Art e storie simili. Il corpo come il linguaggio, Skira editore, Milano 2000.

ZACCURI, Alessandro, "La premiazione. Jean Clair, i titoli tossici dell'arte", Avvenire, 10 Maggio 2014.

\section{Nota biografica}

Serena Feloj è Professore Associato di Estetica presso l'Università degli Studi di Pavia. Ha svolto attività di ricerca presso l'Università degli Studi di Milano e il Max-Planck Institut für empirische Ästhetik di Frankfurt am Main. È stata ricercatrice ospite a Köln, Marburg e Frankfurt am Main e docente ospite a Halle. È autrice delle monografie Il sublime nel pensiero di Kant (Morcelliana, Brescia 2012); Estetica del disgusto. Mendelssohn, Kant e i limiti della rappresentazione (Carocci, Roma 2017); Il dovere estetico. Normatività e giudizi di gusto (Mimesis, Milano 2018). Ha tradotto e curato W. Menninghaus, Disgusto. Teoria e storia di una sensazione forte (Mimesis, Milano 2016) ed è impegnata nella ritraduzione italiana della Fondazione della metafisica dei costumi e della Critica della ragion pratica per Einaudi. 